\title{
Yet Another Real-Time Specification for the Steam Boiler: Local Clocks to Statically Measure Systems Performance ${ }^{\star}$
}

\author{
Candida Attanasio, Flavio Corradini, and Paola Inverardi \\ Dipartimento di Matematica Pura ed Applicata \\ Università dell'Aquila \\ Via Vetoio, Loc. Coppito, L'Aquila, Italy \\ Fax +390862433180 \\ \{flavio, inverard\}@univaq. it
}

\begin{abstract}
In this paper we apply a new real-time modeling to the wellknown Steam Boiler case study. The novelty of our approach is in explicitly representing the various system components, i. e. hardware sensors, software controllers and so on, with separate local clocks. The aim of our approach is to be able to statically analyze the global system specification taking into account the relative speed of each system components. For example, we can statically verify if, and how changing the local speed of a component can affect the global performance of the system. Component behaviors are specified by means of a simple process algebra. Local clocks are modeled as higher order terms in a given signature, and unification is used to define the common clock. Then an operational semantics defines which transitions a process can perform and which transitions let time to elapse.
\end{abstract}

\section{Introduction}

In this paper we apply a new real-time modeling to the well-known "The Steam Boiler" case study taken from [2]. The novelty of our approach is in explicitly representing the various system components, i. e. hardware sensors, software controllers, etc with separate local clocks. The aim of our framework is to be able to statically analyze the global system specification taking into account the relative speed of each system components. For example, we can verify if, and how changing the local speed of a component can affect the global performance of the system. Component behaviors are specified by means of a simple process algebra $[20,15,18]$. Local clocks are modeled as higher order terms in a given signature, and unification is used to define the common clock [16]. Then an operational semantics defines which transitions a process can perform and which transitions let time to elapse similarly to $[7,14,21,10]$.

* This work has been partially funded by CNR, Progetto: Metodologie e strumenti di analisi, verifica e validazione per sistemi software affidabili 
In particular we specify the architecture of a system as a network of (parallel) components, each one with its own local clock. This means that, from the behavioral point of view, we put the components in parallel and let them communicate upon synchronization, similarly to [18]. The global clock of the resulting system is obtained from the local clocks which are modeled as higher order terms in a given signature, through a unification process [16]. If there exists a unifier, all the local clocks will be expressed as suitable linear functions of the global one. Due to the properties of the unification process, i.e. the existence of a unique most general unifier, this clock configuration step represents the best way to relate the local clocks so that the maximum number of synchronizations in the system can happen. The ability of modeling this clock configuration step allows us, besides the usual behavioral and timing analysis, to statically analyze the systems with respect to different properties. For example, we can verify if, and how, changing the local speed (i.e. the local clock) of a component can affect the global performance of the system. That is the amount of synchronizations in the system increases or decreases.

The paper is organized as follows: Section 2, introduces the language of components, and its operational semantics. The language is CCS-like [20] and discusses the use of higher order terms to represent local clocks. Section 3, presents the steam boiler case study. This is a well known and studied example in the literature of which several different specifications exist [19]. Our interest in it is due to the possibility of performing a kind of quantitative analysis which has never been addressed in the previous specifications. Section 4 shows how we can statically reason on the system, that is how we can compare the behavior of different system specifications obtained by considering the same components but with different speed. Section 5 presents conclusions and future works.

\subsection{Related Works}

As far as related works are concerned, in a companion paper, [3] we have extensively motivated this approach in the emerging field of component programming. In fact, besides the traditional field of control systems $[12,2,19]$, the need of assembling together heterogeneous components is more and more frequent due to the widespread diffusion of information technology in any application field, from multimedia applications to telecommunication systems.

The system description language we consider implements several ideas from classic timed process description languages. The idea of using local clocks is taken from $[7,14,10]$, the view of system activities as instantaneous events is taken from [21] (and related works, see references therein). The rational for this choice is that since we are concerned with the static analysis of behavioral aspects of systems obtained by assembling together components running at (possibly) different speed we prefer to abstract from events duration while concentrating on the relative speed of the system components. The relative speed between components, indeed, directly influence the interaction capabilities of the system components which, in turn, may influence the system performance. 
The main difference with these works is that we can model different speed for different system components. This is not possible neither in [21], where the existence of a unique global clock is made explicit (and based upon the fact that all system components must synchronize), nor in $[7,14,10]$, where the local clocks are assumed to elapse in the same way (by making the system components running at the same speed).

The notion of local clocks and a calculus for synchronizing local clocks, is also presented in the language SIGNAL [1,11]. There, a clock is associated with a signal and clocks can be of different frequencies. Although the notion of local clocks and of their calculus might resemble our approach, the use of these notions in the two approaches is different.

\section{The Language and Its Transitional Semantics}

We adopted a two-level syntax which distinguishes between regular processes and interactive processes. Intuitively, a regular process corresponds to a collection of threads of computation which must be performed at a single site. An interactive process corresponds to a (parallel) composition of regular processes which may evolve independently or communicate each other. The language of interactive processes is close to the language presented in [18].

Following [20], we assume a set of actions $A$ (ranged over by $\alpha$ ) from which we obtain the set of co-actions $\bar{A}=\{\bar{\alpha} \mid \alpha \in A\}$ useful to model process synchronizations. We use $A c t$ (ranged over by $a, b, \ldots$ ) to denote $A \cup \bar{A}$, the set of visible actions. The invisible action, useful to model internal activities of process executions, is denoted by $\tau \notin A c t$ and we use $A c t_{\tau}$ (ranged over by $\mu$ ) to denote the set of all actions $A c t \cup\{\tau\} . V$ (ranged over by $x$ ), is the set of process variables used for recursive definitions.

We first define regular processes or $\mathcal{R} \mathcal{P}$ [20] which specify finite, nondeterministic automata familiar from the theory of regular languages. The set of regular processes, $\mathcal{R} \mathcal{P}$ (ranged over by $p, q, \ldots$ ), over $A c t_{\tau}$ and $V$, is the set of closed (i.e., without free variables) generated by the following grammar:

$$
p::=\text { nil } \mid \text { | } p|p| p+p|x| \operatorname{rec} x \cdot p .
$$

Process nil denotes a terminated process. By prefixing a term $p$ with an action $\mu$, we get a process term $\mu . p$ which can perform an action $\mu$ and then behaves like $p . p_{1}+p_{2}$ denotes the non deterministic composition of $p_{1}$ and $p_{2}$, while rec $x . p$ is used for recursive definitions. The standard operational semantics of regular processes is omitted. We just recall few standard notational conventions that will be used in the following. The notation $p[q / x]$, where $p$ and $q$ are terms of the syntax of $\mathcal{R} \mathcal{P}$ terms and $x$ is a process variable, is used to denote the term obtained from $p$ by simultaneously replacing each free occurrence of $x$ by $q$. A transition $p \stackrel{\mu}{\mapsto} p^{\prime}$ says that process $p$ evolves into process $p^{\prime}$ by performing an action $\mu$ (see [20] for its formal definition).

A distributed, interacting process, or $\mathcal{I} \mathcal{P}$ term, is a (finite) parallel combination of regular processes equipped with their own local clocks, or a term restricted 
by an action, or a term relabeled according to a function $\Phi: A_{c} t_{\tau} \longrightarrow$ Act $_{\tau}$ that preserves co-actions and leaves $\tau$ fixed.

Definition 1. (Interacting Processes) The set $\mathcal{I P}$ (ranged over by $P, Q, \ldots$ ) of interacting processes is the least one satisfying the following grammar:

$$
P::=<p, \lambda^{N} x . t^{M}(x)>|P| Q|P \backslash\{a\}| P[\Phi]
$$

where $p \in \mathcal{R} \mathcal{P}, a \in$ Act, $N$ is called degree variable, and $M \in \mathrm{N}^{1}$

Let us spend some words explaining the local clocks and the role they play in analyzing the behavior of concurrent systems. In this paper we model local clocks with a restricted form of the so called "iterative terms" defined in [16]. Iterative terms are higher order terms that finitely represent infinite sequences of first order terms. Thus they seem natural candidates to model time in a discrete way (it can be thought as an infinite sequence of finite objects) and time passing.

Here we make use of a subclass of iterative terms of the form $\lambda^{N} x \cdot t^{m}(x)$, where $m$ is a natural number and $N$ ranges over natural numbers. The intuitive meaning of this term is that by ranging $N$ over natural numbers, we can obtain for each $n$ a term by unfolding the context $t^{m} n$-times at the subterm $x$. Where, by convention, we denote a term $t(t(t(x)))$ with $t^{3}(x)$. For instance, a term like $\lambda^{N} x \cdot t^{2}(x)$ denotes the infinite sequence $\left\{x, t^{2}(x), t^{4}(x), t^{6}(x), \ldots\right\}$. This sequence can be thought as the time instants (ticks) where a process is active and hence can perform an action. It is obtained by letting $N \in\{1,2, \ldots, \omega\} ; N=0$ generates $x$, $N=1$ generates $t^{2}(x), N=2$ generates $t^{4}(x)$, We can think of $x$ as the starting time and of $t^{i}(x)$ as the $i$-th tic of the local clock ( $t$ is a first order term on a certain signature $\Sigma$ ). By varying on the degree variable and assuming different expressions in the body of $\lambda$ iterative terms, we can have a different scale of the time and hence different local clocks (different speeds). For instance term $\lambda^{L} x . t^{3}(x)$ denotes $\left\{x, t^{3}(x), t^{6}(x), t^{9}(x), \ldots\right\}$ and is always obtained by letting $L \in\{1,2, \ldots, \omega\}$.

$\lambda^{N} x \cdot t^{2}(x)$ and $\lambda^{L} x \cdot t^{3}(x)$ with $N$ and $L$ different variables denote the temporal scales of two systems evolving independently according to their own relative speed. The intuition here is that each $\lambda$ iterative term represents an infinite sequence of elements, thus modeling the time sequence.

In this way, we can analyze how a global system behaves when a component is scheduled to be executed on a slower or faster architecture. This amounts at comparing two different systems where the same component has a slower, respectively faster, local clock. For instance a system with local clock $\lambda^{N} x \cdot t^{2}(x)$ is faster than the one with local clock $\lambda^{L} x \cdot t^{3}(x)$ because from the starting time $(x)$ to a common time $\left(t^{6}(x)\right)$ the former local clock has four ticks $\left(\left\{x, t^{2}(x), t^{4}(x), t^{6}(x)\right\}\right)$ and hence the system is able to perform four actions, while the latter has only three $\left(\left\{x, t^{3}(x), t^{6}(x)\right\}\right)$. Common times are particularly interesting to study possible system synchronizations. Two processes can synchronize if they can perform

\footnotetext{
${ }^{1}$ Note that in the syntax of interactive processes, parallelism can only appear at the top level. This ensures us that their standard operational semantics can be described by a finite state transition system.
} 
communicating actions at the same time. Thus, according to the above example, two processes running with speed detected by $\lambda^{N} x . t^{2}(x)$ and $\lambda^{L} x . t^{3}(x)$ respectively, may synchronize (if they can perform communicating actions) at times $\left(\left\{t^{6}(x), t^{12}(x), t^{18}(x), \ldots\right\}\right)$.

Thus common times represent times where process components possibly synchronize. Due to the way we have modeled clocks, the existence of common times depends on the existence of a unifier among all local clocks. The theory of iterative terms states that the problem of determining whether for any pair of iterative terms there exists an (infinite) sequence of common terms or not is decidable. In case it exists a theorem also gives the maximum sequence of common terms (which, intuitively, correspond to the maximum sequence of times where processes can synchronize). In the class of iterative terms we are considering it is always possible to find the maximum sequence of common terms. The theorem gives the most general unifier among terms. The most general unifier relates the degree variables of the iterative terms with a fresh variable degree which generate the maximum sequence of common terms. For instance, the two terms $\lambda^{N} x \cdot t^{2}(x)$ and $\lambda^{L} x \cdot t^{3}(x)$ initially independent unify for $L=2 Q$ and $N=3 Q$ with $Q$ a fresh name. Thus we could also consider iterative terms $\lambda^{3 Q} x . t^{2}(x)$ and $\lambda^{2 Q} x . t^{3}(x)$ to generate the sequence $\left\{x, t^{6}(x), t^{12}(x), t^{18}(x), \ldots\right\}$

The notion of most general unifier is exploited in this paper to detect the least common time (after the starting one) of a given set of iterative terms representing local clocks of parallel processes. This time is the least time where all processes in the net can perform something. The iterative terms then behaves "periodically" with respect to this least time. In the period between the starting time, i.e. the degree variable is $=0$, and the least time, each iterative term can generate different terms. For instance $\lambda^{N} x . t^{2}(x)$ generates $\left\{x, t^{2}(x), t^{4}(x), t^{6}(x)\right\}$ while $\lambda^{L} x . t^{3}(x)$ generates $\left\{x, t^{3}(x), t^{6}(x)\right\}$, where $t^{6}(x)$ is the least time. These terms can be ordered in a standard way: $x<t^{2}(x)<t^{3}(x)<t^{4}(x)<t^{6}(x)$. This ordered sequence gives the finest discrete representation of the time for the two components. At each time of the sequence one of them can perform an action. Being finite, this sequence can be put in bijection with a finite segment of the natural numbers. We can build a matrix of $n$ entries and $m$ columns where $n$ corresponds to the length of the finest sequence from the starting time to the one preceding the least common time and $m$ is the number of parallel regular processes in an interactive term. In our example we have four entries, one for each element of set $\left\{x, t^{2}(x), t^{3}(x), t^{4}(x)\right\}$ and two columns, one for each component.

This matrix indicates which processes are active at a given time, and hence also which processes may engage in a synchronization. To build the matrix, consider the finest (ordered) sequence of terms until the common one and take a term $t$. We put an $X$ at the entry corresponding to $t$ and column corresponding to the process $p$ if the local clock associated with $p$ has term $t$ in its sequence. Clearly we denote with $p$ also every $p$-derivative, that is every state $p$ reaches during a computation. For this reason we will write $M(i, p)=X$ also when $p$ is not exactly the process $p$, denoting a column of the matrix, but a $p$-derivative. 
The matrix corresponding to interactive process

$$
P=<p_{1}, \lambda^{N} x \cdot t^{2}(x)>\mid<p_{2}, \lambda^{M} x \cdot t^{3}(x)>
$$

with $p_{1}$ and $p_{2}$ two regular processes is given in Fig. 1.

Table 1. A Matrix for $P$

\begin{tabular}{|c|c|c|}
\hline & $p_{1}$ & $p_{2}$ \\
\hline$x$ & $X$ & $X$ \\
\hline$t^{2}(x)$ & $X$ & \\
\hline$t^{3}(x)$ & & $X$ \\
\hline$t^{4}(x)$ & $X$ & \\
\hline
\end{tabular}

With abuse of notation, in the rest of this paper, we identify the entries of the matrix (and hence times) with indexes of the matrix itself, i.e. the natural numbers from 0 to $n-1$. We will also say "at time $i$ " to mean the time corresponding to the natural number $i$ in the finest sequence of local times.

Once matrix $M$ is defined, the next step is to show how an interactive process evolves. We can now forget local clocks (they are memorized in the matrix) within interactive processes. To every regular process $p$ we associate a natural number $t$. During the execution of a process which contain $p$ as a component, $t$ says whether $p$ has been active or not at a certain time $i$. In particular, if $p$ has not performed any action at time $i$ then $t=i$, otherwise $t=i+1$. Since, in our setting, time features as relative (not as absolute; i.e., relative to the starting time of the process execution), $t$ will be called a "relative" local clock.

Definition 2. (Processes with Relative Local clocks) The set $S$ (ranged over by $\left.d, d^{\prime}, \ldots\right)$ of processes with relative local clocks is the least one satisfying the following rules:

$$
d::=(p, t)|d| d \mid d \backslash\{a\} \quad d[\Phi]
$$

where $p \in \mathcal{R} \mathcal{P}, t \in \mathbf{N}$ and $a \in$ Act. Clearly, we are only interested in the sublanguage of states $d$ such that every relative local clock appearing within $d$ is in $\{i, i+1\}$ for some $i \in \mathbf{N}$.

We now define the transitional semantics of our $\mathcal{I P}$ processes. This is done by first defining which transitions a process can perform at a given (relative) time $t$ (corresponding to an entry of the matrix) and then defining which transitions let 
time to elapse. The former transitions are given by defining a family of transition relations, one for each entry of the matrix $M$. Let $i$ be an entry of $M$, then a transition, according to the transition relation indexed by $i$, is of the form $d \underset{M}{\stackrel{\mu}{\longrightarrow}} i d^{\prime}$. The inference rules defining this family of transition relations are given in Table 2, where we require for $d \stackrel{\mu}{M} d^{\prime}$ be derivable that every relative local clock within $d$ is in $\{i, i+1\}$.

Table 2. Transitional "at Time" $t$

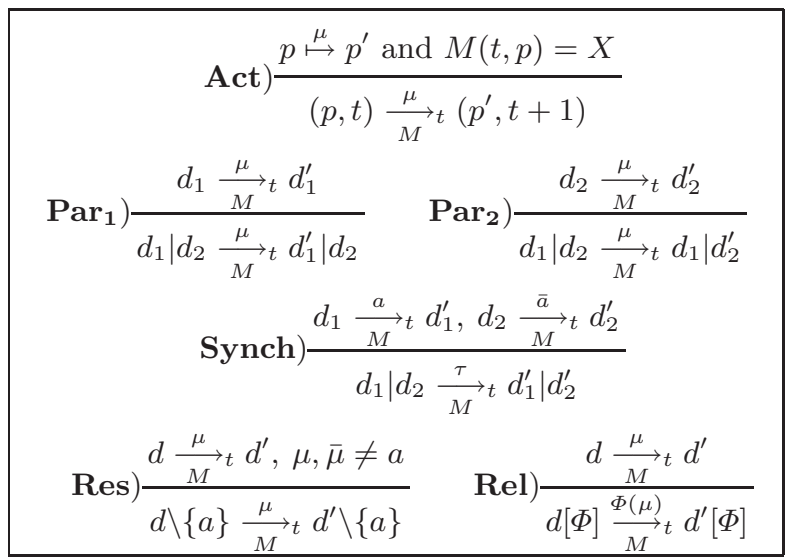

Let us comment the rules in Table 2. The rule for action prefixing Act) states that a regular process $p$ can perform an action $\mu$ at time $t$ leading to a regular process $p^{\prime}$, only if $p$ is active $(M(t, p)=X)$ and $p$ has not already performed any action (its relative local clock $t$, not $t+1$ ) at that time. The relative local clock is set to $t+1$ to remember that it cannot perform any other action at time $t$. Rule $\left.\left.\mathbf{P a r}_{\mathbf{1}}\right)\left(\mathbf{P a r}_{\mathbf{2}}\right)\right)$ for the asynchronous execution of an action $\mu$ from the left (right) subprocess is almost standard. It says that if $d_{1}$ performs an action $\mu$ at time $t$, then the parallel composition $d_{1} \mid d_{2}$ can perform the same action at the same time. Rule Synch) deals with synchronization; two processes can synchronize if and only if they can perform complementary actions at the same time. Rules Res) and $\mathbf{R e l}$ ) for restriction and relabeling are as usual.

Transitions which let time to elapse are of the form $d \stackrel{\mu}{{ }_{M}}{ }_{i} d^{\prime}$. It is worth of note that two subsequent transitions, such as $d \stackrel{\mu}{{ }_{M}}{ }_{i} d^{\prime}$ and $d^{\prime} \underset{M}{\stackrel{\gamma}{\longrightarrow}} d^{\prime \prime}$ may occur at different times (time elapses), while regarding the transition relation $\underset{M}{\stackrel{\mu}{\longrightarrow}} i$ we always have $i=j$.

The inference rules defining the elapsing of time are given in Table 3. There, $u p$ is a function which given a process $d$ and a natural number $n$ updates 
to $n$ every relative local clock appearing within $d$ (e.g., up $((a . p, 2) \mid(b . q, 3), 5)=$ $(a . p, 5) \mid(b . q, 5))$.

When we write $d \underset{M}{\stackrel{\mu}{\longrightarrow}} t$ (instead of $\left.d \underset{M}{\stackrel{\mu}{\longrightarrow} t} d^{\prime}\right)$ we mean that each relative local clock within $d$ is in $\{t, t+1\}$ but there is no $d^{\prime}$ such that $d \underset{M}{\stackrel{\mu}{\longrightarrow}} d^{\prime}$.

Table 3. Modelling the Elapsing of Time

$$
\begin{aligned}
& \text { TheSame) } \frac{d \underset{M}{\stackrel{\mu}{\rightleftarrows} t} d^{\prime}}{d \underset{M}{\stackrel{\mu}{\rightleftharpoons}} t d^{\prime}}
\end{aligned}
$$

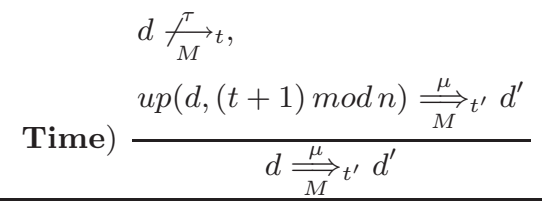

Only rule Time) needs explanation. Assume that process $d$ cannot perform any $\tau$ action at time $t$ while $u p\left(d, t^{\prime}\right)$, where $t^{\prime}=(t+1) \bmod n$ (we set every local clock within $d$ to $t^{\prime}$ in order to figure out which transitions are possible at that time), can perform an action $\mu$ at $t^{\prime}$ leading to $d^{\prime}$. Then also $d$ can perform an action $\mu$ at time $t^{\prime}$ leading to $d^{\prime}$.

The basic idea behind this rule is that synchronizations cannot be delayed. They must be performed as soon as they can. In other words, two processes can synchronize when they perform complementary actions at the same time; if one of the two is able to execute such an action before the other, then a form of busy waiting is allowed. This permits one to model a situation in which a faster process can wait for a slower partner. However, when both partners are ready to synchronize, the handshaking immediately occurs. On the other hand visible actions can be delayed arbitrarily long before firing. This treatment between visible and invisible actions is only apparently different. Invisible actions denote synchronizations between two parallel components of the same process ("internal" synchronizations), while visible actions model synchronizations with the external environment ("external" synchronizations). Thus delayed executions of visible actions models a situation in which the process responsible for their execution is slower (faster) with respect to an hypothetic external faster (slower) partner.

While Section 4 entirely concentrates on the analysis of the systems performance we now show, via a simple example, how the interaction capabilities of the system may influence also its functionality. This is because parallel components can only synchronize at given time instances (detected by the local clocks). 
Hence, some synchronizations might not happen. Consider, for instance, a simple system described by:

$$
S=\left(<p_{1}, \lambda^{N} x \cdot t^{2}(x)>\mid<p_{2}, \lambda^{M} x \cdot t^{4}(x)>\right) \backslash\{b\}
$$

where $p_{1}=\operatorname{rec} x \cdot \tau \cdot(b \cdot x+\tau \cdot x)$ and $p_{2}=\operatorname{rec} x \cdot \bar{b} \cdot x$.

The matrix associated with $M$ is given in Table 4 . It is not difficult to see that

Table 4. A Matrix for System $S$

\begin{tabular}{|c|c|}
\hline$p_{1}$ & $p_{2}$ \\
\hline$X$ & $X$ \\
\hline$X$ & \\
\hline
\end{tabular}

the synchronization on channel $b$ never fires when the local clocks associated with the parallel components $p_{1}$ and $p_{2}$ are $\lambda^{N} x \cdot t^{2}(x)$ and $\lambda^{M} x \cdot t^{4}(x)$, respectively. This phenomenon does not arise if $p_{1}$ and $p_{2}$ are associated, instead, with local clocks generating the same temporal scale. Clearly, as will also be noted in Section 4, this phenomenon does not happen in the class of concurrent but not communicating actions. All actions will eventually be performed.

\section{The Steam Boiler}

The level of water in a boiler, that is heated to generate high-pressure steam, has to be controlled to guarantee certain minimal and maximal water level values (whose violation would damage the steam boiler).

The steam boiler consists of two sensors, MeasS and MeasW, a pump, Pump, a valve, Valve and two controllers, PContr and VContr. The sensor MeasS measures the steam pressure level value within the boiler while the sensor $M e a s W$ measures the water level value. The Pump is used to pump water in the boiler to replace the steam released, and Valve is used to release steam when the pressure within the boiler becomes dangerous. The pump controller PContr switches the pump off when the water reaches the high water level and on when it goes below the low water level. An operator must be informed of any level of the water becoming critical (below the low level water and over the high level water). The valve controller VContr switches the valve on when the steam has to be released and off when the pressure steam level value becomes acceptable. As in the case of the pump controller, an operator must be informed of any level of the steam becoming critical (over the high level steam). See Figure 1 for the software architecture of the steam boiler. 


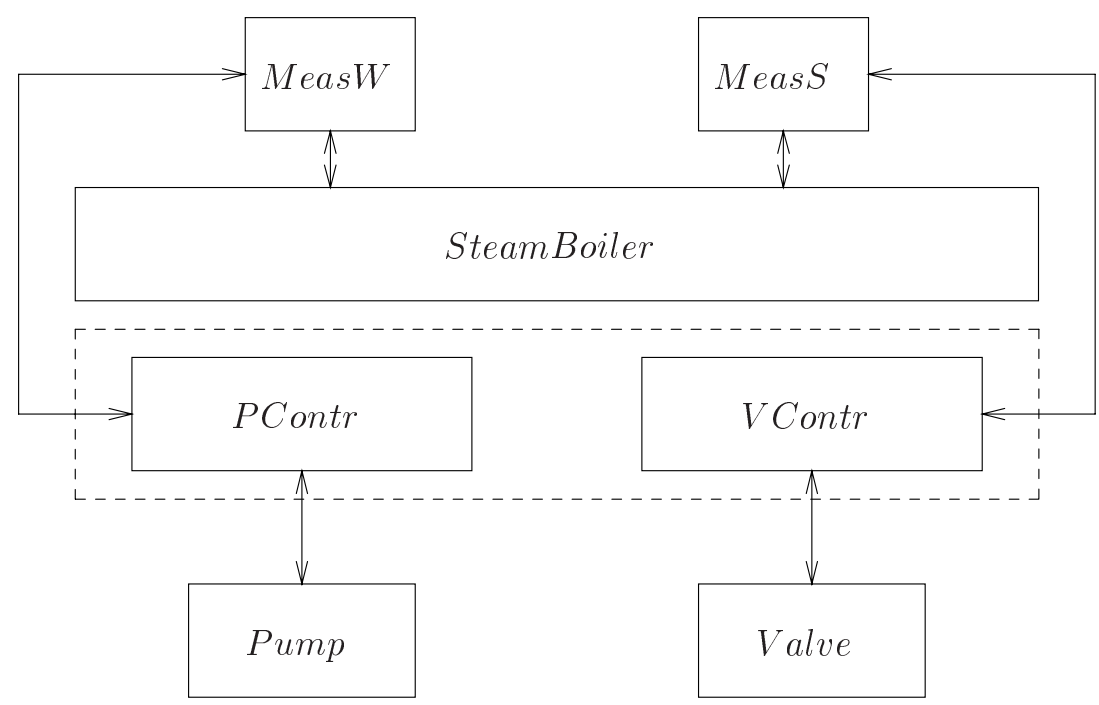

Fig. 1. The Steam Boiler Software Architecture

Let us describe the intended behavior of the sensors. MeasW performs an internal action $\tau$ when the level of water is between the low and high water level. It performs an action LowWater $(l w)$ when the water must be pumped in the boiler and action HighWater $(h w)$ when the actual level of water sufficiently replaces the steam released. When the level of the water is too high or too low $M e a s W$ performs an action DangerWater $(d w)$. Meas $S$ has a similar behavior. It performs an action $\tau$ when the level value of the steam is between the low level and the high level, an action $h s$ when the Valve must be opened (because the level value of the steam is too high), an action $l s$ when the Valve has to be closed and an action $d s$ when the level value of the steam becomes critical (higher than the maximum value allowed).

$$
\begin{aligned}
& \text { Meas } W \equiv \operatorname{rec} x .(\tau . x+h w . x+l w . x+d w . x) \\
& \text { Meas } S \equiv \operatorname{rec} x .(\tau . x+h s . x+l s . x+d s . x)
\end{aligned}
$$

The pump receives pon or poff commands from the pump controller when the water must be pumped in or not respectively.

$$
\text { Pump } \equiv \operatorname{rec} x \cdot(\overline{p o n} \cdot x+\overline{p o f f} \cdot x)
$$

The valve must be opened (closed) when it receives von (voff) commands from the valve controller.

$$
\text { Valve } \equiv \operatorname{rec} x \cdot(\overline{\text { von }} \cdot x+\overline{\text { voff }} \cdot x)
$$


The steam controller is the parallel composition of the pump controller and the valve controller: PContr and VContr. PContr receives signals by MeasW and $V$ ContrE by MeasS.

$$
\text { SContr } \equiv \text { PContr } \mid \text { VContr } .
$$

When PContr receives a LowWater (it communicates with MeasW by performing an action $\overline{l w}$ ) it switches on the pump and when it receives a HighWater (it communicates with Meas $W$ by performing an action $\overline{h w}$ ) it switches off the pump. Finally, if PContr receives a DangerWater level (it communicates with Meas $W$ by performing an action $\overline{d w}$ ) the operator is informed through the execution of an action Emergency. VContr behaves similarly.

$$
\begin{aligned}
\text { PContr } \equiv & \operatorname{rec} x \cdot \overline{d w} \cdot \text { Emergency. } x+ \\
& \operatorname{rec} x \cdot \overline{h w} \cdot \text { poff. } x+ \\
& \operatorname{rec} x \cdot \overline{l w} \cdot \text { pon.x } \\
V \text { Contr } \equiv & \operatorname{rec} x \cdot \overline{d s} \cdot \text { Emergency. } x+ \\
& \operatorname{rec} x \cdot \overline{h s} \cdot \text { von. } x+ \\
& \operatorname{rec} x \cdot \overline{l s} . \text { voff. } x
\end{aligned}
$$

The whole system is the parallel composition of the sensors, the pump, the pump controller, the valve and the valve controller. In our initial configuration, System $_{1}$, we assume that the Valve and the Pump are the faster devices. The sensors MeasW and MeasS are slower than the previous components but faster than the two controllers.

$$
\begin{aligned}
\text { System }_{1} \equiv( & <\text { MeasW, } \lambda^{N} x \cdot t^{3}(x)>\mid \\
& <\text { Meas }, \lambda^{S} x \cdot t^{3}(x)>\mid \\
& <\text { PContr, } \lambda^{R} x \cdot t^{4}(x)>\mid \\
& <\text { VContr, } \lambda^{M} x \cdot t^{4}(x)>\mid \\
& <\text { Pump, } \lambda^{P} x \cdot t^{2}(x)>\mid \\
& \left.<\text { Valve, } \lambda^{Q} x \cdot t^{2}(x)>\right) \backslash\{d w, d s, l w, l s, \text { hw, hs, pon, poff, von, voff }\}
\end{aligned}
$$

The matrix associated with System $_{1}$ is shown in Table 5. The least common time is $t^{12}$ (remember that it is the first time, after the starting time $x$, in the sequence generated by the most general unifier, that is the first time at which all components can perform an action). The finest sequence is

$$
\left\{x, t^{2}(x), t^{3}(x), t^{4}(x), t^{6}(x), t^{8}(x), t^{9}(x), t^{10}(x)\right\} .
$$

These times are denoted by the fragment of natural numbers $\{0,1,2,3,4,5,6,7\}$. We recall that these are all the (local) times at which some component can perform an action.

\section{Analyzing the Steam Boiler Behaviors}

In this section we show how our model can be used to analyze system properties. We are particularly interested in proving properties related to the interaction capabilities of the system components. These may influence the system 
Table 5. A Matrix for System 1

\begin{tabular}{|c|c|c|c|c|c|}
\hline MeasW & MeasS & PContr & VContr & Pump & Valve \\
\hline$X$ & $X$ & $X$ & $X$ & $X$ & $X$ \\
\hline$X$ & $X$ & & & $X$ & $X$ \\
\hline$X$ & $X$ & $X$ & $X$ & $X$ & $X$ \\
\hline$X$ & $X$ & $X$ & $X$ & $X$ & $X$ \\
\hline & & & & & $X$ \\
\hline
\end{tabular}

performance, as we will see in this section, besides the system functionality, as already seen in Section 2. Indeed, the parallel components can only synchronize at given time instances (detected by the local clocks). Hence, some synchronizations might be delayed for a while if not discarded at all.

We can give examples of systems where increasing the speed of a system component always means increasing the performance of the system as a whole. ${ }^{2}$ Consider, for instance, MeasW and MeasS running in parallel:

$$
<\operatorname{Meas} W, \lambda^{R} x \cdot t^{4}(x)>\mid<\operatorname{Meas} S, \lambda^{R} x \cdot t^{4}(x)>.
$$

If we make the temporal scale finer, then the performance of such system always increases. In the case of sensors, this means that more checks are made in the same interval of time. Hence, also means that the danger level water/steam and the high level water/steam are detected sooner.

The slogan "increasing the speed of a system component always means increasing the performance of the system as a whole", however, on one hand is not always desirable, on the other is not always guaranteed. To see that it is not always desirable just think of MeasW and MeasS as video presentation device and sound presentation device in a multimedia system. In this case the two components must proceed together (at the same speed) because it is critical that a sound stream is played out within a reasonable interval of time after/before

\footnotetext{
${ }^{2}$ This is typical in the class of concurrent but non communicating systems.
} 
the video stream is played out. Making a component faster than another one might imply making the presentation of the video and sound streams not "wellsynchronized". In presence of synchronization, moreover, it is not always the case that faster components imply faster systems. Consider, for instance, $(($ MeasW, 0$) \mid($ MeasS, 0$) \mid($ PContr, 0$) \mid($ VContr, 0$) \mid($ Pump, 0$) \mid($ Valve, 0$))$

the initial state of System . $_{\text {. }}$

$$
\backslash\{d w, d s, \text { hw, hs, lw, ls, pon, poff, von, voff }\}
$$

The earlier time the Valve (Pump) is opened in System $_{1}$, due to a HighSteam (LowWater) level, is $t^{4}(x)\left(t^{4}(x)\right)$. This is a case in which the Valve (Pump) runs faster than the Controllers.

As one would expect, if the Valve (Pump) runs slower than the Controllers (replace local clocks $\lambda^{P} x \cdot t^{2}(x)$ and $\lambda^{Q} x \cdot t^{2}(x)$ with $\lambda^{P} x \cdot t^{5}(x)$ and $\lambda^{Q} x \cdot t^{5}(x)$ in System $_{1}$ ) then we would have a worse performance: the Valve (Pump) would be opened at $t^{20}(x)$.

Nevertheless, in some cases, faster components imply worse performances. Consider the following assignment of speeds to the components of our steam boiler system:

$$
\begin{aligned}
\text { System }_{2} \equiv( & <\text { MeasW }, \lambda^{N} x \cdot t^{3}(x)>\mid \\
& <\text { Meas } S, \lambda^{S} x \cdot t^{3}(x)>\mid \\
& <\text { PContr }, \lambda^{R} x \cdot t^{3}(x)>\mid \\
& <\text { VContr, } \lambda^{M} x \cdot t^{3}(x)>\mid \\
& <\text { Pump, } \lambda^{P} x \cdot t^{2}(x)>\mid \\
& \left.<\text { Valve, } \lambda^{Q} x \cdot t^{2}(x)>\right) \backslash\{d w, d s, \text { lw, ls, hw, hs, pon, poff, von, voff }\}
\end{aligned}
$$

where the controllers are faster than the corresponding ones in System Shile $_{1}$ the other components are untouched. We get a matrix with only 4 entries but the least time the Valve (Pump) can be opened, due to a HighSteam (LowWater) level, is time $t^{6}(x)$ (against the $t^{4}(x)$ of System S $_{1}$.

Other interesting properties related to our systems can be analyzed by mapping our abstract interpretation of time and time passing into the common notion of time. This can be done by associating the first order $t$ term, appearing within local clocks, with an interval of real time. Then, every $t^{N}(x)$ (for $N \in \mathbf{N}$ ) denotes a real instant of time for an external observer. If $n$ denotes the number of entries of the actual matrix, the real time associated with $t^{N}(x)$ can be calculated by $N \bmod n$ (the actual entry of the matrix or, in other words, the relative time) and by $N$ div $n$ (how many times the entry of the matrix reaches $n$ and, hence, a new slot of observation is taken into account). Then, always regarding to the steam boiler system, we can answer to safety requirements such as:

- Can the pump be switched on in one hour?

- After how many time units PContr switches on the pump after receiving a LowWater command by MeansW?

- Can the external environment be informed in one hour if the steam boiler gets an emergency state?

- When the controllers receive a DangerWater/DangerSteam which is the least time the environment is informed that the steam boiler gets an emergency state? 
- Are there actions which will never be performed?

- Is our system deadlock-free? And, eventually, which is the least time it will reach a deadlock state?

These questions may have different answers depending on the local clocks chosen to specify the speed of the parallel components, that is depending on the considered configuration.

Moreover, we can prove that the labeled transition system associated with a configuration $d$ is a finite state transition system. This is due to the fact that our systems are interactive processes (which are finite states according to the standard operational semantics [20]) and to the fact that the matrix we are dealing with are finite. Hence, we could use standard tools to generate and to verify the transition systems [8].

\section{Conclusions and Future Work}

In this paper we have applied our approach to model configurations of real-time components to the Steam Boiler case study. Our approach allows a more faithful modelling of systems obtained by assembling together heterogeneous components. The key feature of the approach is the possibility of statically analyze the interaction capabilities of the system components in terms of the possible global system synchronizations. This allows the designer to suitably tune the local speeds of the components, in order to achieve the best performance potentiality of the system. The kind of temporal analysis we have performed on the Steam Boiler example, is completely new with respect to what presented in previous specifications [2] and it is representative of a common need when designing this kind of systems. Very often, in the design of complex systems arises the need of setting the frequency with which a certain component, in our case a sensor but in general any kind of controller, has to exhibit a given event. Associated with the event, there can be a cost (e.g. a transmission cost), thus it is mandatory for the designer to choose the right local timing which maximizes the global performance while minimizing costs.

The $\lambda$ iterative terms we used in this paper were very simple and did not show the $\lambda$ iterative terms potentiality. We expect to better exploit the $\lambda$ iterative terms expressive power in modeling, besides time, other quality parameters of heterogeneous system components, like for example frequency or capacity. Future works are in the direction of applying our approach to different kind of systems for which some kind of tuning of quality parameters should be done.

\section{References}

1. Amagbegnon,T.P., Besnard,L., Le Guernic,P.: Implementation of the data-flow Synchronous Language Signal. Proc. ACM Symposium on Programming Languages design and ImplementationACM (1995) 163-173. 47

2. Formal Methods for Industrial Applications. Abrial,J-R., Börger,e., Landmaack,H. Eds., Springer Verlag (1996). 45, 46, 58 
3. Attanasio,C., Corradini,F., Inverardi,P.: Static Analysis of Real-Time Componentbased Systems Configurations, Internal Report 11/98, Universita' dell'Aquila (1998). 46

4. Allen,R., Garlan,D.: A Formal Basis for Architectural Connection. ACM Transactions on Software Engineering and Methodology, 6(3) (1997) 213-249.

5. Blair,G.S., Blair,L., Bowman,H., Chetwynd,A.: Formal Specification in Distributed Multimedia Systems. University College London Press (1997).

6. Bowman,H., Faconti,G., Katoen,J-P., Latella,D., Massink,M.: automatic Verification of a Lip Synchronization Algorithm Using UPPAAL. In FMICS'98 (1998).

7. Aceto,L., Murphy,D.: Timing and Causality in Process Algebra. Acta Informatica 33(4) (1996), 317-350. 45, 46, 47

8. Cleaveland,R., Parrow,J., Steffen,B.: The concurrency workbench: A semanticsbased tool for the verification of concurrent systems. In ACM Transaction on Programming Languages and Systems 15 (1993). 58

9. Coen-Porisini,A., Ghezzi,C., Kemmere,R.A.: Specification of Realtime Systems Using ASTRAL. In IEEE Transaction on Software Engineering 23 (9) (1997).

10. Corradini,F.,: On Performance Congruences for Process Algebras. Information and Computation 145 (1998), 191-230. 45, 46, 47

11. Project EP-ATR. Rapport d'activite' scientifique 1997. IRISA, INRIA-Rennes (1997). 47

12. Friesen,V., Jähnichen,S., Weber,M.: Specification of Software Controlling a Discrete-Continuos Environment. ACM Proceedings ICSE97 (1997), 315-325. 46

13. Ghezzi,C., Mandrioli,D., Morzenti,A.: TRIO: A Logic Language for Executable Specifications of Real-Time Systems. Journal Systems and Software, 25 (2) (1994).

14. Gorrieri,R., Roccetti,M., Stancampiano,E.: A Theory of Processes with Durational Actions. Theoretical Computer Science 140(1) (1995), 73-94. 45, 46, 47

15. Hoare,C.A.R.: Communicating Sequential Processes. Prentice-Hall, Englewood Cliffs, New Jersey (1985). 45

16. Intrigila,B., Inverardi,P., Venturini Zilli,M.: A Comprehensive Setting for Matching and Unification over Iterative Terms. submitted to Fundamenta Informaticae. 45, 46,48

17. Inverardi,P., Wolf,A.L.: Formal Specification and Analysis of Software Architectures using the Chemical Abstract Machine Model. IEEE Transactions on Software Engineering, 21(4) (1995), 373-386.

18. Kramer,J., MageeJ.: Exposing the skeleton in the coordination closet. Proc. of Coordination'97, LNCS 1282, Springer-Verlag (1997), 18-31. 45, 46, 47

19. Real-Time Systems Specification, Verification and Analysis. Mathai,J. Ed., Prentice Hall Internation Series in Computer Science (1996). 46

20. Milner,R.: Communication and concurrency. International series on computer science, Prentice Hall International (1989). 45, 46, 47, 58

21. Moller,F., Tofts,C.: A Temporal Calculus of Communicating Systems. Proc. of CONCUR'90, LNCS 459, Springer-Verlag (1990), 401-415. 45, 46, 47 